\title{
CDISC SDTM Arm Null Reason Response Terminology
}

National Cancer Institute

\section{Source}

National Cancer Institute. CDISC SDTM Arm Null Reason Response Terminology. NCI

Thesaurus. Code C142179.

Terminology associated with the arm null reason response codelist of the Clinical Data

Interchange Standards Consortium (CDISC) Study Data T abulation Model (SDT M). 